\title{
Contexto Familiar Relacionado a las Habilidades Sociales de las y los Adolescentes. Distrito Gregorio Albarracín. Tacna 2010
}

\author{
Family Context Related to the Social Skills and Teens. \\ Albarracín Gregorio District. Tacna 2010
}

'María Dalila Salas Romero, ${ }^{2}$ María del Carmen Silva Cornejo

\begin{abstract}
RESUMEN:
El presente estudio de investigación se realizó con la finalidad de determinar la relación entre el contexto familiar y las habilidades sociales de las y los adolescentes del distrito Gregorio Albarracín Lanchipa. En el estudio la muestra estuvo constituida por 80 escolares adolescentes hombre y mujeres de 14 a 18 años elegidos al azar y que estudian en instituciones educativas del distrito en mención. Para ello se utilizó el test de valoración de habilidades sociales (MINSAPERU) y el test para valorar el contexto familiar a través del funcionamiento familiar: Entre los principales resultados obtenidos tenemos que el mayor porcentaje de los adolescentes percibe un contexto familiar regular (80\%) y presenta una categoria promedio de habilidades sociales: Autoestima, comunicación asertividad y toma de decisiones $(28.75 \%)$. La mayoria de adolescentes pertenece a familias mucleares (68\%). Estadisticamente no existe relación significativa entre el contexto familiar y las habilidades sociales de las y los adolescentes.
\end{abstract}

Palabras clave: adolescente, familia, habilidades sociales, contexto familiar:

\section{ABSTRACT:}

This research study was done in order to determine the relationship between family background and social skills of adolescents Gregorio Albarracin district. The study sample consisted of 80 school teenage men and women (14 to 18 year old) randomly selected and studied in educational institutions in the district mentioned. For this a Social Skills valorative test was used (MINSAPERU) and the test to assess the family context through family functioning. The main results obtained; the highest percentage of teens perceives a regular family context $(80 \%)$ and presents an average category of social skills: self-esteem, assertiveness communication and making decisions $(28.75 \%)$. Most of teenagers belong to muclear families $(68 \%)$. Statistically, there is no significant relationship between family background and social skills of adolescents.

Keywords: adolescent, family. social skills, family context.

\footnotetext{
'Maestra en Enfermeria, Enfermera, Licenciada en Enfermeria. Facultad de Ciencias de la Salud. Universidad Nacional Jorge Basadre Grolımann

"Maestra en Enfermeria, Licenciada en Enfermeria. Facultad de Ciencias de la Salud. Universidad Nacional Jorge Basadre Grohınann
} 


\section{INTRODUCCIÓN}

Las Habilidades Sociales han sido tratadas por numerosos autores en diferentes escuelas, uno de los máximos exponentes es Solter, reconocido como padre de la terapia de conducta, quién en 1949 introdujo en término basado en la necesidad de aumentar la expresividad de los individuos, algunas de las sugerencias son utilizadas en la actualidad. Desde esa fecha a la actualidad han surgido disímiles criterios que distan más o menos del primero, sin embargo se mantiene la esencia donde se encuentran exponentes como Wolpe (1958), Alberti \& Emmens (1978). Linehan (1984), Phillips (1985), Curran (1985), Argyle \& kedon (1987) y finalmente Caballo quién en 1987 emite un criterio con la cual la mayor parte de los investigadores trabajan en la actualidad el tema. Los trabajos se basan en la realización de un E.H.S (Entrenamiento en Habilidades Sociales) la cual esta matizada por un conjunto de técnicas o momentos especificos que la ayudan a desarrollar el déficit de H.S en áreas y vida de un individuo, o problemática que se desencadena a raiz del intercambio social, sin embargo no existe referencia que permita valorar elementos internos o externos que actúen como determinantes en la formación de habilidades sociales.

En un entrenamiento en habilidades sociales realizado por Martínez Negreira \& Sanz Martinez (2001) en la ciudad de Santiago de Cuba a un grupo de jóvenes se pudo apreciar que existian elementos que entorpecian o facilitaban el desarrollo de habilidades tan necesarias para un exitoso desempeño en la sociedad actual, donde las exigencias de hoy son superiores a las de ayer e inferiores a las de mañana.

Por ello este trabajo pretende valorar elementos que actúan como determinantes del desarrollo exitoso o no de habilidades sociales (autoestima. asertividad, Comunicacion y toma de decisiones).

Es en este sentido el entorno familiar juega un papel importante en el desarrollo de habilidades sociales adecuadas que permitiran al adolescente relaciones sociales satisfactorias tanto con su pares como con otros grupos generacionales. haciendo uso apropiado de conductas asertivas, comunicación eficaz, autoestima y toma de decisiones adecuadas y oportunas.

Para conocer aquellos aspectos familiares que pueden favorecer o entorpecer el desarrollo del niño se consideran los siguientes indicadores: Estructura familiar, relaciones familiares, ayuda en la enseñanza de proceso aprendizaje, estructura de valores familiares y relación familia escuela. Lo que nos planteamos fue responder a la siguiente interrogante: ¿Existe relacion entre el Contexto familiar y las habilidades sociales de las y los adolescentes del Distrito Gregorio Albarracin Lanchipa?

\section{Objetivos}

Determinar la relación entre contexto familiar y las habilidades sociales de las y los adolescentes del distrito

\section{Gregorio Albarracin Lanchipa.}

Identificar la percepción del contexto familiar de las y los adolescentes del estudio.

Determinar el nivel de habilidades sociales de las y los adolescentes del estudio.

Determinar las categorias promedio de habilidades sociales de los y las adolescentes (asertividad. comunicación, autoestima, toma de decisiones).

\section{MATERIALYMÉTODOS}

El estudio desarrolló el Tipo de Investigación Descriptivo Correlacional que nos permitió arribar a conclusiones especificas que serán de utilidad para una intervención oportuia. La muestra estuvo constituida por 80 escolares adolescentes hombres y mujeres de 14 a 18 años. La recolección de datos se realizó utilizando dos instrumentos, una entrevista estructurada para determinar algunas caracteristicas socioculturales del adolescente, un test de valoración de habilidades sociales. (MINSA-PERU), test para evaluar contexto familiar a través funcionamiento familiar.

La información obtenida se proceso mediante el paquete estadístico SPSS y se presenta en tablas de frecuencia de una o dos entradas, para el análisis estadistico se empleo el Test de independencia de Criterios de Chi Cuadrado.

\section{RESULTADOS}

Tabla N ${ }^{\circ} 01$. Distribución de Adolescentes según habilidades sociales. Distrito Gregorio Albarracin.

Tacna 2010

\begin{tabular}{lcc}
\hline \multicolumn{3}{c}{ Habilidades Sociales } \\
Categorias & $\mathrm{N}^{\circ}$ & $\%$ \\
\hline Muy bajo & 2 & 2.5 \\
Bajo & 11 & 13.75 \\
Promedio bajo & 23 & 28.75 \\
Promedio & 20 & 25 \\
Promedio alto & 21 & 26.25 \\
Alto & 3 & 3.75 \\
Muy alto & 80 & 100 \\
Total & & \\
\hline
\end{tabular}

Fuente: Encuesta Aplicada

Interpretación:

En la presente tabla podemos observar que el $28.75 \%$ de las y los adolescentes presentan una calegoria promedio de habilidades sociales. seguida de una categoria alta con un $26.25 \%$. 
Tabla $\mathbf{N}^{\circ}$ 02. Distribución de Adolescentes Según Habilidades Sociales: Asertividad Distrito Gregorio Albarracin, Tacna 2010

\begin{tabular}{|c|c|c|}
\hline \multicolumn{3}{|c|}{ Asertividad } \\
\hline Categorias & $\mathrm{N}^{\circ}$ & $\%$ \\
\hline \multicolumn{3}{|l|}{ Muy Bajo } \\
\hline Bajo & 4 & 5 \\
\hline Promedio Bajo & 9 & 11.25 \\
\hline Promedio & 19 & 23.75 \\
\hline Promedio Alto & 21 & 26.25 \\
\hline Alto & 18 & 22.5 \\
\hline Muy Alto & 9 & 11.25 \\
\hline Total & 80 & 100 \\
\hline
\end{tabular}

Fuente: Encuesta aplicada

Interpretación:

En cuanto a la habilidad social: Asertividad observamos que las y los adolescentes presentan categoría en promedio alto con un $26.25 \%$ seguido de categoria promedio con un 23.75 .

Tabla $\mathbf{N}^{\circ}$ 03. Distribución de Adolescentes según Habilidades Sociales: Comunicación Distrito Gregorio Albarracin, Tacna 2010

\begin{tabular}{lcc}
\hline \multicolumn{3}{c}{ Comunicación } \\
Categorias & $\mathrm{N}^{\circ}$ & $\%$ \\
\hline Muy Bajo & 2 & 2.5 \\
Bajo & 23 & 28.75 \\
Promedio Bajo & 30 & 37.5 \\
Promedio & 15 & 18.75 \\
Promedio Alto & 5 & 6.25 \\
Alto & 1 & 1.25 \\
Muy Alto & & 100 \\
\hline
\end{tabular}

Fuente: encuesta aplicada

Interpretación:

En el presente tabla observanios que en la habilidad social: Comunicación las y los adolescentes presentan categoría promedio con un $37.5 \%$, seguido de la categoria promedio bajo con un $28.75 \%$.
Tabla $\mathbf{N}^{\circ}$ 04. Distribución de Adolescentes Según Habilidades Sociales: Autoestima Distrito Gregorio Albarracin, Tacna 2010

\begin{tabular}{|c|c|c|}
\hline \multicolumn{3}{|c|}{ Autoestima } \\
\hline Categorias & $\mathrm{N}^{\circ}$ & $\%$ \\
\hline \multicolumn{3}{|l|}{ Muy Bajo } \\
\hline Bajo & 2 & 2.5 \\
\hline Promedio Bajo & 7 & 8.75 \\
\hline Promedio & 23 & 28.75 \\
\hline Promedio Alto & 28 & 35 \\
\hline Alto & 15 & 18.75 \\
\hline Muy Alto & 5 & 6.25 \\
\hline Total & & 100 \\
\hline
\end{tabular}

Fuente: Encuesta aplicada

Interpretación:

En cuanto a la habilidad social: Autoestima, observamos que las y los adolescentes presentan un $35 \%$ en la categoría promedio alto, y un $28.75 \%$ en la categoria promedio.

Tabla № 05. Distribución de Adolescentes Según Habilidades Sociales: Toma de Decisiones Distrito Gregorio Albarracín. Tacna 2010

\section{Toma de Decisiones}

\begin{tabular}{lcc}
\multicolumn{1}{r}{ Categorías } & $\mathrm{N}^{\circ}$ & $\%$ \\
\hline Muy Bajo & & \\
Bajo & & \\
Promedio Bajo & 9 & 11.25 \\
Promedio & 16 & 20 \\
Promedio Alto & 30 & 37.5 \\
Alto & 18 & 22.5 \\
Muy Alto & 7 & 8.75 \\
Total & & 100 \\
\hline
\end{tabular}

Fuente: Encuesta aplicada

Interpretación:

En el Tabla $\mathrm{N}^{\circ} 05$ observamos que en la habilidad social: Toma de decisiones el $37.5 \%$ presenta una categoría promedio alto y un $22.5 \%$ categoria alto. 
Tabla $\mathbf{N}^{\circ}$ 06. Distribución de Adolescentes según Percepción de Contexto Familiar. Distrito Gregorio Albarracín. Tacna 2010.

\begin{tabular}{lcc}
\hline \multicolumn{3}{c}{ Contexto Familiar } \\
\multicolumn{1}{c}{ Rango } & $\mathrm{N}^{\circ}$ & Porcentaje \\
\hline Bueno & 4 & $3.0 \%$ \\
Regular & 67 & $88.0 \%$ \\
Bajo & 9 & $9.0 \%$ \\
Total & 80 & $100 \%$ \\
\hline
\end{tabular}

Fuente: Encuesta aplicada

\section{Interpretación:}

De acuerdo a la Tabla 6 se puede evidenciar del $100 \%$ de adolescentes encuestados, el $88 \%$ tiene una percepción regular del contexto familiar, el $9 \%$ percibe un contexto familiar bajo y el $3 \%$ percibe un contexto familiar bueno.

De acuerdo a la Tabla 7 se puede evidenciar que del $100 \%$ de adolescentes encuestados, el mayor porcentaje que percibe un contexto familiar regular presenta habilidades sociales promedio con un $28.4 \%$ y promedio alto con un $26.9 \%$. En cuanto al grupo de adolescentes que percibe un contexto familiar bueno presenta habilidades sociales nivel alto con un $75 \%$. Estos datos sometidos a análisis estadístico con la prueba de independencia de criterios chi cuadrado no guardan relación significativa entre variables.

Tabla N 07. Distribución de Adolescentes Según Contexto Familiar y Habilidades Sociales. Distrito Gregorio Albarracín, Tacna 2010

\begin{tabular}{lcccccc}
\hline \multicolumn{1}{c}{ Habilidades Sociales } & \multicolumn{2}{c}{ Bueno } & \multicolumn{2}{c}{$\begin{array}{c}\text { Contexto Familiar } \\
\text { Regular }\end{array}$} \\
\cline { 3 - 7 } \multicolumn{1}{c}{ Categorias } & $\mathrm{N}^{\circ}$ & $\%$ & $\mathrm{~N}^{\circ}$ & $\%$ & $\mathrm{~N}^{\circ}$ & $\%$ \\
\hline Muy Bajo & 0 & $0.0 \%$ & 0 & $0.0 \%$ & 0 & $0.0 \%$ \\
Bajo & 0 & $0.0 \%$ & 2 & $3.0 \%$ & 0 & $0.0 \%$ \\
Promedio Bajo & $\mathrm{I}$ & $25.0 \%$ & 9 & $13.4 \%$ & 1 & $11.1 \%$ \\
Promedio & 0 & $0.0 \%$ & 19 & $28.4 \%$ & 4 & $44.4 \%$ \\
Promedio Alto & 0 & $0.0 \%$ & 18 & $26.9 \%$ & 2 & $22.2 \%$ \\
Alto & 3 & $75.0 \%$ & 16 & $23.9 \%$ & 2 & $22.2 \%$ \\
Muy Alto & 0 & $0.0 \%$ & 3 & $4.5 \%$ & 0 & $0.0 \%$ \\
Total & 4 & $100.0 \%$ & 67 & $100.0 \%$ & 9 & $100.0 \%$ \\
\hline
\end{tabular}

Tabla 8. Prueba de Chi-Cuadrado

\begin{tabular}{lccc}
\hline $\begin{array}{l}\text { Prueba de Chi- } \\
\text { Cuadrado }\end{array}$ & Valor & gl & $\begin{array}{c}\text { Sig. } \\
\text { asintótica } \\
\text { (bilateral) }\end{array}$ \\
\hline $\begin{array}{l}\text { Chi-cuadrado } \\
\text { de Pearson }\end{array}$ & 8.255 & 10 & .604 \\
$\begin{array}{l}\text { Razón de } \\
\text { verosimilitudes }\end{array}$ & 9.812 & 10 & .457 \\
$\begin{array}{l}\text { Asociación } \\
\text { lineal por lineal }\end{array}$ & .688 & 1 & .407 \\
$\begin{array}{l}\text { N de casos } \\
\text { válidos }\end{array}$ & 80 & & \\
\hline
\end{tabular}

\section{DISCUSIÓN}

La adolescencia es en general una etapa de desarrollo personal, fisiológico, social y emocional rápido. Incluye aprender y experimentar la sexualidad y varias formas de relaciones humanas, así como el desarrollo de la auto identidad y la autoestima. Para un número cada vez mayor de adolescentes, es una época en la que se reciben grandes presiones de los padres. las madres. los medios de comunicación.

El proceso de socialización se lleva en primer lugar por la familia quien inicia el proceso para la formación de habilidades sociales, lo que continúa en la escuela donde se enfatiza y obliga a desarrollar las habilidades más complejas, la formación de estas habilidades implica las importantes relaciones que establezca con el grupo de amigos y otros de su entorno.

Las edades de los adolescentes que participaron en la muestra oscilan entre 14 a 18 años, la mayoria residentes de la zona (Gregorio Albarracín Lanchipa), el 68\% pertenecian a famiiias nucleares. el 12\% vivian con algún familiar (tía, abuela, hermano mayor), el $20 \%$ pertenece a una familia extensa. La mayoría de adolescentes eran alumnos regulares y su edad concordaba con el año cursado. Como se puede ver los porcentajes del tipo de familia concuerdan con la estructura familiar del pais, en donde la mayoría pertenece a una familia nuclear. 
En el presente trabajo de investigación no se encontró relación significativa entre el contexto familiar y las habilidades sociales de las y los adolescentes, conclusión arribada después que los datos fueron sometidos a análisis estadistico a través de la prueba de independencia de criterios chi cuadrada.

Sin embargo Martínez (2001), encontró relación entre la influencia familiar y las habilidades sociales de adolescentes que asistian a la preparatoria, donde detalla a los factores que favorecen o entorpecen este proceso, tal como la estructura familiar, las relaciones familiares, actitud de la familia ante los resultados escolares, estructura de valores familiares.

Torres Velásquez y Rodriguez Soriano (2006) en un estudio realizado en México, sobre contexto familiar de los estudiantes universitarios en relación con su rendimiento académico, encontraron que existe relación entre estas dos variables y demuestran la importancia que tiene incluir a la familia para elevar y promover el rendimiento académico.

Aspectos importantes que se deben trabajar con los padres según Norman (2004) son las categorías contextuales familiares como: amor, trato positivo, autoridad y tiempo, que conllevarán a relaciones sociales satisfactorias en sus hijos, lo que demuestra, que el contexto familiar va repercutir en el manejo eficaz de sus habilidades sociales.

\section{V.CONCLUSIONES}

Con la finalidad de determinar la relación que existe entre el contexto Familiar y las habilidades sociales del adolescente, se realizó el presente estudio de tipo descriptivo, a fin de obtener información que permita elaborar programas dirigidos a incluir a la familia en el trabajo con las y los adolescentes llegando a las siguientes conclusiones:

- No existe Relación significativa entre el Contexto familiar y el nivel de habilidades sociales del adolescente.

- El mayor porcentaje de adolescentes percibe un contexto familiar regular.

- En su mayoría las y los adolescentes presenta una categoría promedio de habilidades sociales.

- El mayor porcentaje de adolescentes presenta asertividad promedio alto.
- El mayor porcentaje de adolescentes presenta comunicación promedio.

- El mayor porcentaje de adolescentes presenta autoestima promedio alto.

- El mayor porcentaje de adolescentes presenta toma de decisiones promedio alto.

- La mayoría de adolescentes pertenece a familias nucleares $(68 \%)$.

- Las edades promedio de los y las adolescentes oscila entre 16 y 18 años.

\section{REFERENCIAS BIBLIOGRÁFICAS}

Bustamante S. (2004). Enfermeria Familiar: Principios del cuidado a partir del saber común de las familias. Perú UNT.

Darley, Glucksberg, Kinchla (2000) "Psicología" I ra. Edición, Ed. Hispanoamericana, Mexico.

MINSA Manual de habilidades sociales en adolescentes escolares. Dirección General de Salud de las Persona". Perú 2006.

Medellin Gladys (1995) "Crecimiento y Desarrollo del Ser Humano". Edit.Huadalupe Colombia

Palacios (2003)," La Familia como Contexto de Desarrollo Humano", A lianza editorial, Madrid 2003.

OPS/OMS. (2002) La familia su dinámica y tratamiento. Ediciones PALTEX. Estados Unidos, 2002.

Shaffer David ( 2000) "Psicologia del Desarrollo" Edit. International Thomson. Mexico 2000.

Torrez Velaasquez, Rodriguez Soriano (2004) "Rendimiento Académico y Contexto Familiar en Estudiantes Universitarios" México.

Correspondencia:

Maria Dalila Salas Romero

Ciudad Universitaria Fundo "Los Granados"

Av. Miraflores s/nt. Tacna. Perú

msalasr@unjbg.edu.pe 